Note

\title{
Quantitative Analysis of Yellow Pigment in Takuan-zuke and Their ABTS Radical
}

\section{Cation Scavenging Activity}

\author{
Asaka Takahashi ${ }^{1}$, Hiroki Matsuoka ${ }^{2 *}$, Emi Watanabe $^{2}$, Atsuko Shinagawa $^{2}$, Yoshio Ozawa $^{2}$, Yoichi Yamada ${ }^{3}$ and \\ Yasushi $\mathrm{UDA}^{4}$ \\ ${ }^{1}$ Nagano Prefectural College, 8-49-7 Miwa, Nagano 380-8525, Japan \\ ${ }^{2}$ Department of Health and Nutrition, Takasaki University of Health and Welfare, 37-1 Nakaorui-cho, Takasaki-shi, Gunma 370-0033, \\ Japan \\ ${ }^{3}$ Faculty of Education, Utsunomiya University, 350 Mine-machi, Utsunomiya-shi, Tochigi 321-8505, Japan \\ ${ }^{4}$ Department of Bioproductive Sciences, Faculty of Agriculture, Utsunomiya University, 350 Mine-machi, Utsunomiya-shi, Tochigi \\ 321-8505, Japan
}

Received November 5, 2008; Accepted February 7, 2009

2-[3-(2-Thioxopyrrolidin-3-ylidene)methyl]-tryptophan (TPMT) is the main yellow pigment in salted radish roots (takuan-zuke). In the present study, we investigated the content of 1-(2-thioxopyrrolidin-3yl)-1,2,3,4-tetrahydro- $\beta$-carboline-3-carboxylic acid (TPCC) as a precursor of the yellow pigment and TPMT in commercial takuan-zuke. The total content of stereoisomers was 0.2-36.4 mg/100 g (2-364 ppm) for TPCC, and 0-6.6 mg/100 g (0-66 ppm) for TPMT. TPCC and TPMT showed radical scavenging activity against ABTS radical cation than the classical antioxidant (ascorbic acid and Trolox). The antioxidative activity showed no differences for TPCC stereoisomers, but showed differences for $E / Z$ ratiocontrolled TPMT solutions.

Keywords: yellow pigment, tryptophan derivative, tetrahydro- $\beta$-carboline derivative, salted radish root, radical scavenging activity

\section{Introduction}

Takuan-zuke, the salted or dried radish root that is pickled in salty rice bran, is a traditional food in Japan. It was originally defined as the processing radish that is now called genboku, a raw material used in various pickled products. Takuan-zuke now refers to genboku that has been further pickled in seasoning liquid and is classified into hoshi-takuan and shio-oshi-takuan depending on the initial method of processing the radish. Hoshi-takuan is first dried and pickled in salty rice bran paste and then further pickled in seasoning liquid. Shio-oshi-takuan is compressed and salted, and then further pickled in seasoning liquid. In addition, Sato-shiboridaikon is the one that salted radish was pickled in seasoning liquid containing liquid sugar syrup.

The radish root turns yellow during the several months

*To whom correspondence should be addressed.

E-mail:matsuoka@takasaki-u.ac.jp of the salting and fermenting processes. Yellowing effects increase particularly in summer, and the generated yellow pigment is related to radish pungent component, 4-methylthio-3-butenyl isothiocyanate (MTBITC) (Ozawa et al., 1990c). MTBITC is mainly converted to 2-thioxo3-pyrrolidinecarbardehyde (TPC) in the presence of water (Uda et al., 1990), with 1-(2-thioxopyrrolidin-3-yl)-1,2,3,4tetrahydro- $\beta$-carboline-3-carboxylic acid (TPCC) formed as a precursor through the Pictet-Spengler reaction with L-tryptophan (Ozawa et al., 1990a, Ozawa et al., 1990b). We previously reported that TPCC is unstable under neutral $\mathrm{pH}$ and is easily converted into $(E)$-2-[3-(2-thioxopyrrolidin-3-ylidene)methyl]-tryptophan ((E)-TPMT) as the main yellow pigment (Matsuoka et al., 2002). (E)-TPMT was isomerized to (Z)-TPMT by long wave UV irradiation, and the isomerization from $(Z)$ - to $(E)$-form is induced by Vislight irradiation (Matsuoka et al., 2008). The color of (Z)TPMT is a darker yellow than $(E)$-TPMT and fades easily 
by Vis-light irradiation, indicating the important role of (Z)TPMT in the yellow color of takuan-zuke. Figure 1 shows the formation of the yellow pigment from MTBITC.

Tetrahydro- $\beta$-carbolines occur in many foods and exhibit a broad range of pharmacological and biological activity (Herraiz, 2000). They can function as neurotransmitters and neuromodulators (Buckholtz, 1980). Recently, Herraiz and Galisteo reported that tetrahydro- $\beta$-carbolines in foods and biological systems act as radical scavengers in the 2,2'-azinobis-(3-ethylbenzothioazoline-6-sulfonic acid) (ABTS) radical cation assay (Herraiz and Galisteo, 2002). L-Tryptophan is an essential amino acid for humans, and as the precursor of the neurotransmitter serotonin, it regulates physiological mechanisms. Several tryptophan derivatives also function as free radical scavengers and antioxidants, stimulating a number of antioxidative enzymes and stabilizing cell membranes (Reiter et al., 1999). Thus, TPCC and TPMT may be applied for uses other than is current use as a pigment. As only mutagenic and antimutagenic properties of TPCC and TPMT have been reported (Ozawa et al., 1999, Matsuoka et al., 2002), we investigated the amount of TPCC and TPMT in commercial takuan-zuke and their radical scavenging activities using an ABTS radical cation assay.

\section{Materials and Methods}

Materials ABTS and Trolox (6-hydroxy-2,5,7,8-tetramethylchroman-2-carboxylic acids) were purchased from Sigma-Aldrich Co. (St. Louis, MO, USA). $N^{\alpha}$-(5-Fluolo2,4-dinitrophenyl)-L-alaninamide (L-FDAA) and (+)-catechin were obtained from Tokyo Chemical Industry Co., Ltd. (Tokyo, Japan). Potassium persulfate was purchased from Kanto Chemical Co., Inc. (Tokyo, Japan). MTBITC was prepared from radish sprouts according to our previously described method (Uda et al., 1990).

Quantitative analysis of TPCC and TPMT in commercial takuan-zuke All takuan-zuke samples (hoshi-takuan, shiooshi-takuan, and sato-shibori-daikon) and ggaktugi (cubed radish kimchi) were purchased from a local market; ggaktugi is a radish sprinkled with chili peppers and pickled in salt, seasoning, and fish sauce. TPCC and TPMT were extracted from each sample as described previously (Matsuoka et al., 2002) and then analyzed by HPLC with using an eluent of acetonitrile: $25 \mathrm{mM}$ phosphate buffer $(\mathrm{pH} \mathrm{6.6)}(18: 82, \mathrm{v} / \mathrm{v})$ for $6 \mathrm{~min}$; flow rate was kept constant at $0.6 \mathrm{~mL} / \mathrm{min}$ and the column temperature was $40^{\circ} \mathrm{C}$. TPCC and TPMT were detected at $268 \mathrm{~nm}$ and $380 \mathrm{~nm}$, respectively.

To separate the TPMT enantiomers extracted from salted radish (genboku), derivatization by L-FDAA (Marfey and Ottesen, 1984) was done. The mixture of $50 \mu \mathrm{L}$ of $0.5 \%$ L-FDAA (in acetone), $100 \mu \mathrm{L}$ of $1 \mathrm{M}$ sodium bicarbonate,
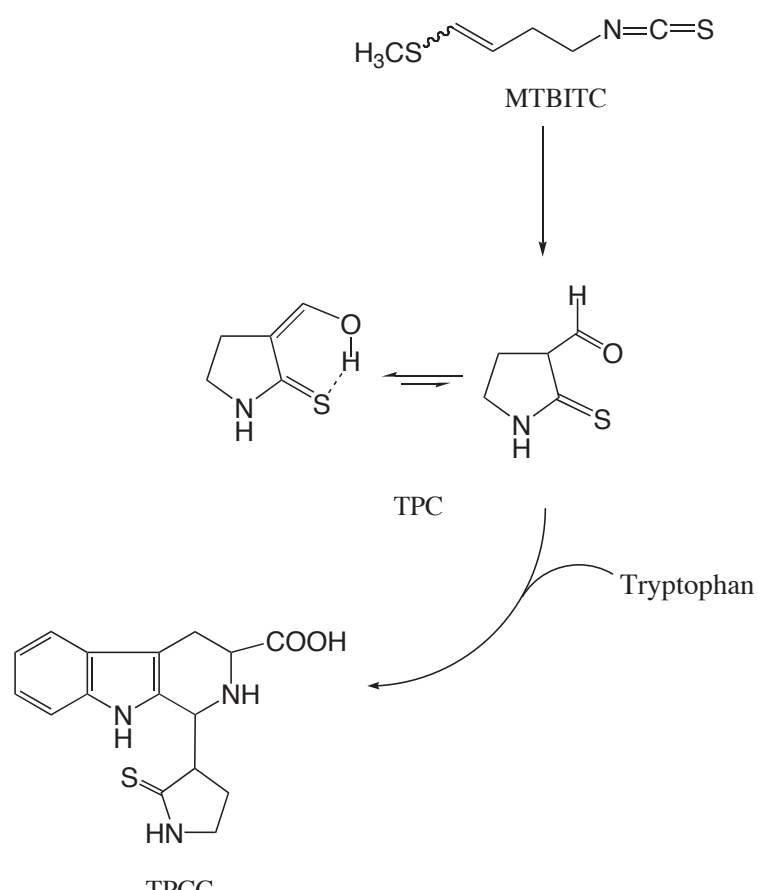

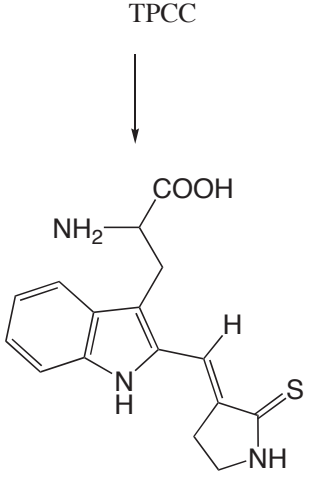

(E)-TPMT

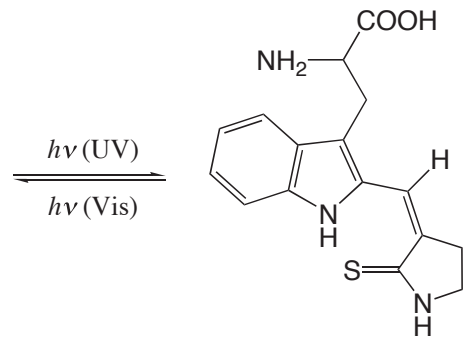

(Z)-TPMT
Fig. 1. Formation of yellow pigment from MTBITC.

and sample solution $(100 \mu \mathrm{L})$ was incubated for $60 \mathrm{~min}$ at $40^{\circ} \mathrm{C}$. The reaction was terminated by the addition of $100 \mu \mathrm{L}$ of $1 \mathrm{M} \mathrm{HCl}$, and the samples were then diluted with $475 \mu \mathrm{L}$ methanol. These solutions were analyzed by HPLC and LCMS. Analytical HPLC was performed with Agilent series 1100 instrument with a photodiode array detector and a Mightysil RP-18 column $(150 \times 3 \mathrm{~mm}$ I.D., Kanto Chemical Co., Ltd., Tokyo, Japan). The mobile phase was composed of acetonitrile (Solvent A) and $25 \mathrm{mM}$ ammonium acetate buffer ( $\mathrm{pH}$ 6.6, Solvent B). Elution was performed with a linear gradient from $15 \%$ to $35 \%$ Solvent A for $30 \mathrm{~min}$. The column temperature was maintained at $45^{\circ} \mathrm{C}$ and the flow rate was $0.4 \mathrm{~mL} / \mathrm{min}$. Derivatives of TPMT were detected at $340 \mathrm{~nm}$.

LC-MS analysis was performed using an Agilent HPLCMSD series 1100 instrument. MS analysis was carried out by electrospray ionization (ESI) in the positive mode. The desolvation temperature was $350^{\circ} \mathrm{C}$, and the capillary volt- 
age was $3.5 \mathrm{kV}$. Nitrogen was used as the desolvation and nebulizer gas.

Preparation of TPCC and TPMT TPCC was synthesized from MTBITC and L- or D-tryptophan according to the method described previously (Ozawa et al., 1999). The diastereoisomeric mixture of $\left(1 S, 3 S, 3^{\prime} R\right)$ - and $\left(1 R, 3 S, 3^{\prime} R\right)$-TPCC was subsequently separated by preparative ODS-HPLC. TPMT derived from TPCC (diastereoisomeric mixture) was prepared according to our previously described method (Matsuoka et al., 2002). The enantiomers of synthesized TPMT were analyzed as described above.

ABTS radical cation scavenger test The effect of TPCC and TPMT on the ABTS radical cation $\left(\mathrm{ABTS}^{\circ+}\right)$ was estimated according to the method of Re et al. (1999). ABTS was dissolved in ultra pure water to a final concentration of 7 $\mathrm{mM}$ and the $\mathrm{ABTS}^{\cdot+}$ was produced by reacting stock solution with potassium persulfate (final conc., $2.4 \mathrm{mM}$ ). The mixture was allowed to stand at room temperature overnight $(>12$ h) in the dark. The ABTS $^{\circ+}$ solution was diluted with $8 \mathrm{mM}$ phosphate buffered saline (PBS) to give an absorbance value of 0.70 at $734 \mathrm{~nm}$. The assay was done using a 96-well microplate. After addition of $200 \mu \mathrm{L}$ diluted $\mathrm{ABTS}^{*+}$ solution to $1 \mu \mathrm{L}$ test compounds or Trolox standard in $70 \%$ methanol, absorbance was measured using a Corona MTP-800Lab microplate reader $90 \mathrm{sec}$ after initial mixing. All determinations were carried out at least three times. The antioxidant capacity was measured in comparison with Trolox, $(+)$-cat- echin, and ascorbic acid as the antioxidant standard.

To compare the radical scavenging activity of geometrical isomers of TPMT, $E / Z$ ratio-controlled solutions were also examined. The TPMT solution (100 ppm) in phosphate buffer ( $\mathrm{pH}$ 5.0) was exposed to visible light at 460 $\mathrm{nm}$ for $15 \mathrm{~min}$ (Solution A). Solution A was re-irradiated at $370 \mathrm{~nm}$ (Solution B). These solutions were prepared with bubbling nitrogen. Irradiation was carried out using the excitation monochromator of a Shimadzu RF-1500 spectrofluorometer (Kyoto, Japan). From the HPLC analysis, the $E / Z$ ratio of Solutions A and B was 96:4 and 0.95:1, respectively (Matsuoka et al., 2008). In test tube, the reaction mixture contained ABTS ${ }^{\cdot+}$ solution, Solution A or Solution B $(0-23.81 \mu \mathrm{M})$ and $70 \%$ methanol at a final volume of $1 \mathrm{~mL}$. The mixture was allowed to react for $15-90 \mathrm{sec}$ after initial mixing. The absorbance was measured using a Hitachi U-2000 double-beam spectrophotometer.

Molecular modeling method Calculation of the lowest energy conformer was done using conformer plug-in in the Marvin suite academic package (version 5.1.03, Chemaxon, Budapest, Hungary) on an Apple MacBook Pro computer running MacOS 10.5.6.

\section{Results and Discussion}

The amounts of TPCC and TPMT in takuan-zuke samples are presented in Table 1 . In our previous study (Matsuoka et al., 2002), we found that salted rad-

Table 1. Amounts of TPCC and TPMT of takuan-zuke samples.

\begin{tabular}{|c|c|c|c|}
\hline \multirow{2}{*}{ Sample } & \multicolumn{3}{|c|}{ Amounts (mg / $100 \mathrm{~g}$ ) } \\
\hline & $\left(1 S, 3 S, 3^{\prime} R\right)$-ТРCC & $\left(1 R, 3 S, 3^{\prime} R\right)$-ТPCC & TPMT $^{\mathrm{a}}$ \\
\hline Hoshi-takuan 1 & $17.13 \pm 0.05$ & $5.21 \pm 0.00$ & $3.43 \pm 0.02$ \\
\hline Hoshi-takuan 2 & $17.17 \pm 0.30$ & $4.89 \pm 0.09$ & $2.94 \pm 0.09$ \\
\hline Hoshi-takuan 3 & $24.85 \pm 0.35$ & $7.31 \pm 0.18$ & $5.58 \pm 0.68$ \\
\hline Hoshi-takuan 4 & $28.29 \pm 0.37$ & $8.13 \pm 0.15$ & $3.43 \pm 0.12$ \\
\hline Hoshi-takuan 5 & $20.06 \pm 0.18$ & $6.86 \pm 0.01$ & $1.34 \pm 0.36$ \\
\hline Hoshi-takuan 6 & $1.71 \pm 0.26$ & $1.52 \pm 0.22$ & $6.55 \pm 0.04$ \\
\hline Hoshi-takuan 7 & $17.67 \pm 0.21$ & $4.71 \pm 0.05$ & $2.73 \pm 0.02$ \\
\hline Hoshi-takuan 8 & $7.86 \pm 0.04$ & $3.36 \pm 0.02$ & $4.96 \pm 0.00$ \\
\hline Shio-oshi-takuan 1 & $9.71 \pm 0.20$ & $2.76 \pm 0.03$ & $0.81 \pm 0.03$ \\
\hline Shio-oshi-takuan 2 & $1.20 \pm 0.03$ & $0.31 \pm 0.00$ & $0.05 \pm 0.00$ \\
\hline Shio-oshi-takuan 3 & $4.85 \pm 0.08$ & $1.77 \pm 0.08$ & $0.38 \pm 0.03$ \\
\hline Shio-oshi-takuan 4 & $2.48 \pm 0.01$ & $0.47 \pm 0.00$ & $0.33 \pm 0.27$ \\
\hline Shio-oshi-takuan 5 & $0.70 \pm 0.04$ & $0.02 \pm 0.03$ & n.d. \\
\hline Sato-shibori-daikon 1 & $1.32 \pm 0.02$ & $0.02 \pm 0.03$ & n.d. \\
\hline Ggaktugi 1 & $0.93 \pm 0.01$ & $0.14 \pm 0.00$ & n.d. \\
\hline Ggaktugi 2 & $1.29 \pm 0.02$ & $0.33 \pm 0.01$ & n.d. \\
\hline Ggaktugi 3 & $2.16 \pm 0.03$ & $1.44 \pm 0.06$ & n.d. \\
\hline Ggaktugi 4 & $0.16 \pm 0.08$ & n.d. & n.d. \\
\hline
\end{tabular}

TPCC, 1-(2-thioxopyrrolidin-3-yl)-1,2,3,4-tetrahydro- $\beta$-carboline-3-carboxylic acid; TPMT, 2-[3-(2-thioxopyrrolidin-3-ylidene) methyl]-tryptophan; n.d.: Not detected.

Values are expressed as means $\pm \mathrm{SD}$.

${ }^{\text {a }}$ The total amount of $E$ - and $Z$-form. 
ish roots (genboku) contained about $50 \mathrm{ppm}$ TPCCs $\left(\left(1 S, 3 S, 3^{\prime} R\right)\right.$-TPCC: $\left(1 R, 3 S, 3^{\prime} R\right)$-TPCC $\left.=4: 1\right)$ and about 6 ppm TPMT. In the commercial takuan-zuke, the content of $\left(1 S, 3 S, 3^{\prime} R\right)$ - and $\left(1 R, 3 S, 3^{\prime} R\right)$-TPCCs as precursors of the yellow pigment was $0-8.1 \mathrm{mg} / 100 \mathrm{~g}(0-81 \mathrm{ppm})$ and $0.2-28.3 \mathrm{mg} / 100 \mathrm{~g}(2-283 \mathrm{ppm})$, respectively. The ratio of $\left(1 S, 3 S, 3^{\prime} R\right)$-TPCC to $\left(1 R, 3 S, 3^{\prime} R\right)$-TPCC was about $3: 1$. TPMT, the main yellow pigment, contained $0-6.6 \mathrm{mg} / 100 \mathrm{~g}$ (0-66 ppm). On average, the amount of TPCCs in hoshitakuan was about 5-fold that in shio-oshi-takuan, while the amount of TPMT was 13-fold. Thus, shio-oshi-takuan appears to contain scarce amounts of TPCCs and TPMT, most likely because it is manufactured from a desalinated salted radish (genboku). In contrast, ggaktugi contained only TPCC and no TPMT, indicating that the amount of TPCC is related to the level of fermentation with lactic acid bacteria.

As the L-isomer of amino acids is known to elute prior to the D-isomer, based on an analysis of L-FDAA derivatives (Fujii et al., 1997), we analyzed TPMT as the analogue of tryptophan. TPMT synthesized from MTBITC and L-tryptophan resulted in only the L-isomer. Similarly, only D-TPMT was generated from D-tryptophan. Taken together with previous results (Ozawa et al., 1999), TPCCs gener- ated from MTBITC and L-tryptophan afford $\left(1 S, 3 S, 3^{\prime} R\right)$ and $\left(1 R, 3 S, 3^{\prime} R\right)$-isomer. In contrast, TPMT extracted from salted radish roots (genboku) afforded only the L-form. As commercial takuan-zuke is made from salted radish roots (genboku), only the L-form of TPMT in commercial takuanzuke is expected to be obtained.

The activity of TPCC and TPMT synthesized from MTBITC and tryptophan as radical scavengers was measured with the ABTS radical cation assay. TPCC and TPMT showed antioxidant activity that was stronger than the classical antioxidant ascorbic acid and Trolox, but weaker than catechin (Fig. 2). Linear curves were obtained with concentrations of the compounds lower than $10 \mu \mathrm{M}$, suggesting that the reaction in not significantly affected by possible side reactions. No difference was observed in the activity of $\left(1 S, 3 S, 3^{\prime} R\right)$ - and $\left(1 R, 3 S, 3^{\prime} R\right)$-TPCC or in the activity of either the enantiomers of tryptophan or TPMT. The antioxidant activity of samples was expressed as Trolox equivalent antioxidant capacity (TEAC) $(\mu \mathrm{M})$, which represents the concentration $(\mu \mathrm{M})$ of Trolox, with the same activity as $1 \mu \mathrm{M}$ of the sample. Similar antioxidant values of TEAC ranging from 2.52 to $2.68 \mu \mathrm{M}$ were obtained for tryptophan and TPMT. The TEAC value of tryptophan and TPMT was

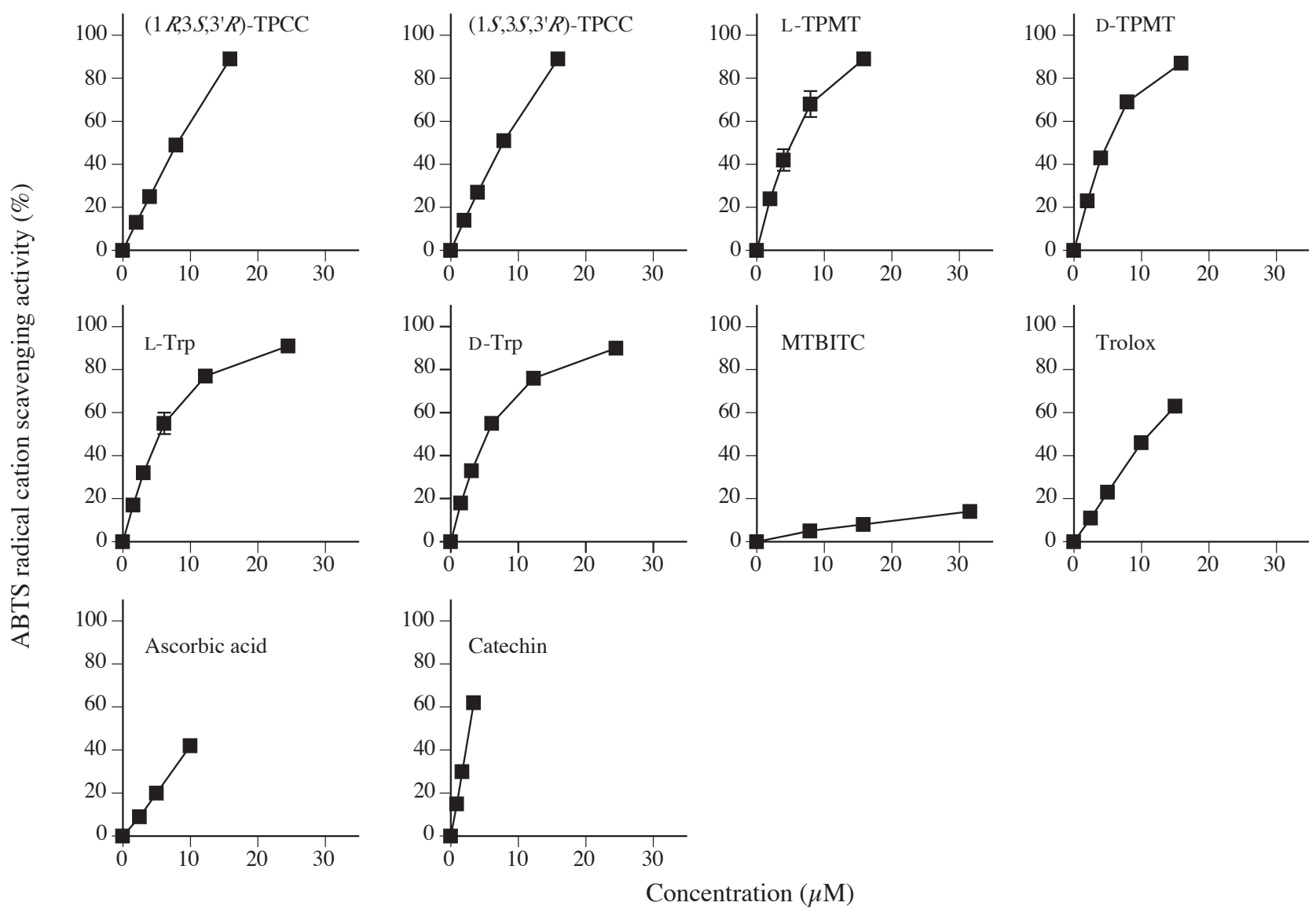

Fig. 2. ABTS radical cation scavenging activity of TPCC, TPMT, and classical antioxidants. Results are expressed as means \pm SD. 


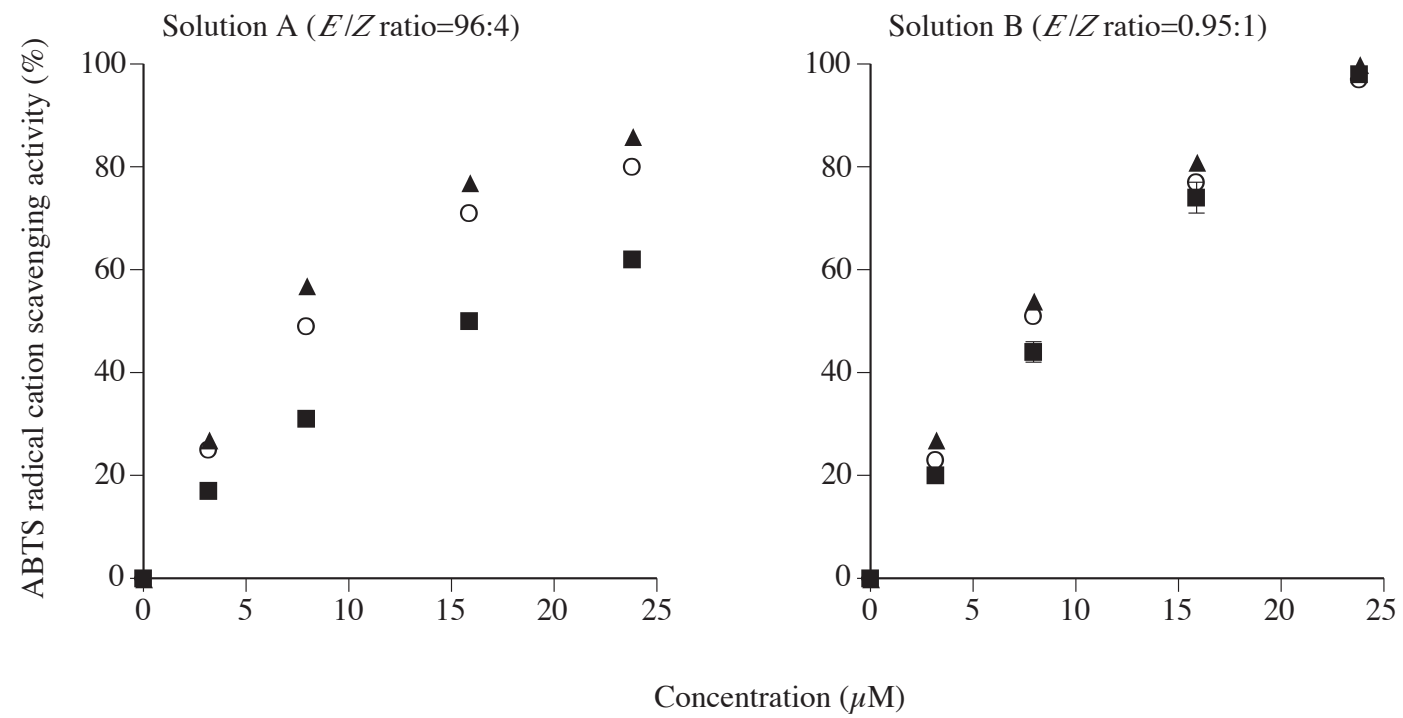

Fig. 3. ABTS radical cation scavenging activity of geometrical isomers of TPMT. ABTS ${ }^{\circ+}$ solution and TPMT reacted for $15 \mathrm{sec}(\boldsymbol{\square}), 60 \mathrm{sec}(\bigcirc), 90 \mathrm{sec}(\boldsymbol{\Delta})$ after initial mixing. Results are expressed as means $\pm \mathrm{SD}$.

higher than that of ascorbic acid $(0.93 \mu \mathrm{M})$ and Trolox, and were $75 \%$ of catechin $(3.45 \mu \mathrm{M})$. The TEAC value of TPCC was $1.48-1.61 \mu \mathrm{M}$ and about $50 \%$ of catechin. MTBITC showed hardly any antioxidant activity against the ABTS ${ }^{\circ+}$. Tryptophan is converted to kynurenine and $\mathrm{N}$-formylkynurenine through the cleavage of indole ring by hydroxyl radical attack or one-electron oxidation (Maskos et al., 1992), and tetrahydro- $\beta$-carboline carboxylic acids are decarboxylated to afford the fully aromatic compounds after reaction with the $\mathrm{ABTS}^{\circ+}$ (Herraiz and Galisteo, 2002), suggesting antioxidative ability of TPMT and TPCC.

Most of TPMT prepared from TPCC by our previously described method was the $E$-form. (E)-TPMT is isomerized to (Z)-TPMT by long wave UV irradiation. To compare the radical scavenging activity of these geometrical isomers, the effect of $E / Z$ ratio-controlled solutions (Solutions A and $\mathrm{B}$ ) on the $\mathrm{ABTS}^{\circ+}$ was examined. The radical scavenging activity of Solution B was higher than that of Solution A; this remarkable activity remained for a short time (Fig. 3). Thus, (Z)-TPMT appears to react with $\mathrm{ABTS}^{\circ+}$ earlier than (E)-TPMT. Calculation of the lowest energy conformer of TPMT showed that indole and thioxopyrrolidine of the $(E)$ TPMT molecule were a coplanar structure (Matsuoka et al., 2002), and the energy of $E$ - and $Z$-forms was calculated to be $56.36 \mathrm{kcal} / \mathrm{mol}$ and $59.69 \mathrm{kcal} / \mathrm{mol}$, respectively. These results suggested that the reactivity of (Z)-TPMT to ABTS ${ }^{\circ+}$ is higher than that of $(E)$-TPMT.

The formation of the (Z)-TPMT contributes to yellowing of takuan-zuke, even though this isomer is more sensitive to light irradiation than the $E$-isomer (Matsuoka et al., 2008). $E$-isomerization of (Z)-TPMT under visible light irradiation may therefore cause partial discolorization of the commercial takuan-zuke. Moreover, as some of the $\beta$-carbolines are accumulated in human tissues, offering protection against oxidative stress (Pari et al., 2000), TPCC and TPMT might also prevent deterioration in the quality of takuan-zuke by oxidation and exhibit antioxidant activity in human tissues.

Acknowledgement We are grateful to Mr. H. Karasawa (Nagano Prefecture General Industrial Technology Center) for his support of the LC-MS analysis.

\section{References}

Buckholtz, N. S. (1980). Neurobiology of tetrahydro- $\beta$-carbolines. Life Sci., 27, 893-903.

Fujii, K., Ikai, Y., Mayumi, T., Oka, H., Suzuki, M. and Harada, K. (1997). A nonempirical method using LC/MS for determination of the absolute configuration of constituent amino acids in a peptide: elucidation of limitations of Marfey's method and of its separation mechanism. Anal. Chem., 69, 3346-3352.

Herraiz, T. (2000). Analysis of the bioactive alkaloids tetrahydro$\beta$-carboline and $\beta$-carboline in food. J. Chromatogr. A, 881, 483-499.

Herraiz, T. and Galisteo, J. (2002). Tetrahydro- $\beta$-carboline alkaloids that occur in foods and biological systems act as radical scavengers and antioxidants in the ABTS assay. Free Radic. Res., 36, 923-928.

Marfey, P. and Ottesen, M. (1984). Determination of D-amino acids. I. Hydrolysis of DNP-L-amino acid methyl esters with carboxypeptidase-Y. Carlsberg Res. Commun., 49, 585-590.

Maskos, Z., Rush, J. D., and Koppenol, W. H. (1992). The hydroxylation of tryptophan. Arch. Biochem. Biophys., 296, 514-520. 
Matsuoka, H., Honzawa, S., Takahashi, A., Yoshikawa, H., Watanabe, E., Watanabe, T., Ozawa, Y., Yamada, Y., Iizuka, T. and Uda, Y. (2008). Photoisomerization of 2-[3-(2-thioxopyrrolidin3 -ylidene)methyl]-tryptophan, a yellow pigment in salted radish roots. Biosci., Biotechnol., Biochem., 72, 2262-2268.

Matsuoka, H., Takahashi, A., Ozawa, Y., Yamada, Y., Uda, Y. and Kawakishi, S. (2002). 2-[3-(2-Thioxopyrrolidin3 -ylidene)methyl]-tryptophan, a novel yellow pigment in salted radish roots. Biosci. Biotech. Biochem., 66, 1450-1454.

Ozawa, Y., Kawakishi, S., Uda, Y. and Maeda, Y. (1990a). Isolation and identification of a novel $\beta$-carboline derivative in salted radish roots, Raphanus Sativus L. Agric. Biol. Chem., 54, 1241-1245.

Ozawa, Y., Uda, Y. and Kawakishi, S. (1990b). Generation of a $\beta$-carboline derivative, the yellowish precursor of processed radish roots, from 4-methylthio-3-butenyl isothiocyanate and L-tryptophan. Agric. Biol. Chem., 54, 1849-1851.

Ozawa, Y., Uda, Y., Matsuoka, H., Abe, M., Kawakishi, S. and Osawa, T. (1999). Occurrence of Stereoisomers of 1-(2'-pyrrolidinethione-3'-yl)1,2,3,4-tetrahydro- $\beta$-carboxylic acid in fermented radish roots and their different mutagenic properties. Biosci.,
Biotechnol., Biochem., 63, 216-219.

Ozawa, Y., Uda, Y., Ohshima, T., Saito, K. and Maeda, Y. (1990c). Formation of yellow pigment by the reaction of 4-methylthio3-butenyl isothiocyanate with L-ascorbic acid and some dihydroxyphenolic compounds. Agric. Biol. Chem., 54, 605-611.

Pari, K., Sundari, C. S., Chandani, S. and Balasubramanian, D. (2000). $\beta$-Carbolines that accumulate in human tissues may serve a protective role against oxidative stress. J. Biol. Chem., 275, 2455-2462.

Re, R., Pellegrini, N., Proteggente, A., Pannala, A., Yang, M. and Rice-Evans, C. (1999). Antioxidant activity applying an improved ABTS radical cation decolorization assay. Free Radic. Biol. Med., 26, 1231-1237.

Reiter, R. J., Tan, D. X., Cabrera, J. and D’Arpa, D. (1999). Melatonin and tryptophan derivatives as free radical scavengers and antioxidants. Adv. Exp. Med. Biol., 467, 379-387.

Uda, Y., Ozawa, Y., Ohshima, T. and Kawakishi, S. (1990). Identification of enolated 2-thioxo-3-pyrrolidinecarbardehyde, a new degradation product of 4-methylthio-3-butenyl isothiocyanate. Agric. Biol. Chem., 54, 613-617. 